\title{
Definición de un modelo de clima laboral basado en la gestión por competencias
}

\author{
Ángel José Olaz Capitán \\ Universidad de Murcia \\ Departamento de Sociología y Política Social \\ Campus universitario de Espinardo. 30100 Murcia \\ olazcapi@um.es
}

\section{Resumen}

El análisis del clima laboral responde a una visión multivariante en la que, desde la década de los años 60 del pasado siglo, diferentes investigadores lo han modelizado evidenciando diferentes dimensiones explicativas de la realidad socio-organizativa. En este sentido, y desde nuestra particular percepción del fenómeno - la correspondencia existente entre clima laboral y la redefinición de un modelo de gestión por competencias- se aporta un nuevo enfoque de análisis que confiamos ayude a explicar el comportamiento esperado de las organizaciones.

Palabras clave: adaptación, flexibilidad, agentes del cambio, análisis de problemas, toma de decisiones, apreciación personal del individuo, competencias, comunicación efectiva, contenido del trabajo, contexto organizativo, clima laboral, iniciativa, proactividad, significado del grupo, trabajo en equipo.

\section{Abstract. Model definition of labour climate based on competencies management}

The analysis of the labour climate is related to a multivariant vision in which, from the 60's decade of the last century, different investigators have shaped it showing different explanatory dimensions of the socio-organizational reality. In this respect, and from our particular perception of the phenomenon - the existing correspondence between labour climate and the redefinition of a model of management by competences - the contribution is a new approach of analysis that we entrust it will help to explain the expected behavior of the organizations.

Key words: adjustment, flexibility, change agents, problem analysis, decisions working, personal appraisal of the individual, competences, effective communication, work content, organizational context, labour climate, meaning of the group, team work

\section{Sumario}

1. Introducción

2. Aproximaciones a la definición del concepto de clima laboral

3. El papel de los protagonistas

4. Dimensiones propuestas para la medición del clima laboral
5. Papel de la gestión por competencias en la gestión del clima laboral

6. Conclusiones

Bibliografía 


\section{Introduccion}

Una atenta mirada a los múltiples estudios orientados a la medición del clima laboral en las organizaciones, constata notables diferencias entre las distintas investigaciones realizadas; en parte, debidas a las dificultades de partida para conceptualizar este término, así como el siempre controvertido alcance y proyección que presenta sobre el individuo, el grupo y la organización.

Sin ánimo de plantear una discusión teórica acerca de cuál es el modelo metodológico más efectivo -algo tan ocioso como inútil, debido a que los enfoques pueden ser complementarios-, el objetivo principal de esta nota de investigación quiere reflexionar sobre la medición del clima laboral de las organizaciones, desde la perspectiva que brindan las competencias profesionales de los trabajadores.

Dicho de otra forma, analizar en qué modo la organización dotada de un modelo competencial coherente, puede intervenir más y mejor en el clima laboral esperado.

\section{Aproximaciones a la definición del concepto de clima laboral}

Debido a la dificultad formal que supone enunciar el concepto de clima laboral, se recurrirá a un conjunto de rasgos que ayuden a aproximar su significado:

- Es un conjunto de variables situacionales de distinto orden y naturaleza, que oscilan en el transcurso del tiempo, afectando de desigual manera a todos aquellos miembros de la comunidad laboral.

- Responde a una lógica de continuidad, ya que es una manifestación de las inercias culturales de la organización, aunque éstas puedan variarse, con independencia del nivel de esfuerzo requerido para ello.

- Está condicionado por dos dimensiones, la interna (la propia organización) $y$, en alguna medida, la externa (entorno con el que interactúa la organización, por ejemplo: clientes, competidores, proveedores, etcétera).

- Está determinado en su mayor parte por las características, las conductas, las actitudes, las aptitudes, las expectativas y, cómo no, por las realidades sociológicas, económicas y culturales de la empresa.

- Es un fenómeno «exterior» al individuo, aunque vivenciado en primera persona, del que se puede valer para amplificar los efectos sobre sí mismo, el grupo en que éste se desenvuelve y la propia organización a la que pertenece.

En otras palabras y de manera más intuitiva, el clima laboral tiene que ver con las sensaciones, no siempre generalmente compartidas por los miembros de un equipo humano y que se recogen bajo otros términos más cotidianos como el «ambiente», la «atmósfera» o el «aire» que se respira. 


\section{El papel de los protagonistas}

Antes se mencionó una dimensión interna y externa del clima laboral en las organizaciones. La relevancia de cada uno de estos dos elementos es clave en la comprensión del fenómeno.

Del mismo modo que nadie cuestiona cómo el propio trabajador puede contribuir a una mejora, a un mantenimiento o a una degradación del clima laboral, es razonable entender que otros agentes externos - como clientes, competidores y proveedores, entre otros-, a través de sus actitudes y comportamientos colectivos, influyen en la salud del clima laboral. Por ejemplo, clientes insatisfechos pueden causar frente común, lo cual repercute en el entorno laboral del trabajador.

Debido a lo inabarcable de estos colectivos, la generalidad de los estudios de clima centran sus investigaciones de "puertas adentro», centrándose en los siguientes protagonistas:

- El individuo, concebido como el sujeto que responde a dos necesidades: por un lado, a impulsar sus aptitudes personales desaprovechadas en su puesto de trabajo y, por otro, al desarrollo de su rol laboral.

- El grupo, entendido como un conjunto de individuos hipotéticamente orientados hacia un mismo fin concreto. Si la variable de referencia trasciende el concepto hacia el de grupo informal, la riqueza adicional es mayor, dado que entonces se tratarán de satisfacer las necesidades sociales que se producen en el puesto de trabajo y en el tiempo libre.

- La organización, entendida como el marco institucional que recoge las necesidades de los grupos y de los individuos involucrados en una estrategia concreta.

\section{Dimensiones propuestas para la medicion del clima laboral}

En un intento por mostrar una visión complementaria a los tradicionales modelos explicativos ${ }^{1}$, lo que sigue a continuación se configura en torno a cuatro escenarios que ayudarían a medir el clima laboral de una organización, son los siguientes: el contexto organizativo, el contenido del trabajo, el significado del grupo y la apreciación personal del individuo, cada uno de éstos con su particular resonancia:

- Contexto organizativo: recoge aquellas variables emparentadas con la estructura organizativa en su más amplio nivel. Ello requiere, al menos, hablar

1. De entre los estudios realizados, destacan, por sus contribuciones teóricas, las aportaciones de Halpin, A. y Crofts, D. (1963); Forehand, G. y Gilmer, B. (1964); Likert, R. (1967); Meyer, H. H. (1968); Schneider, B. y Bartlett, C. J. (1968); Friedlander, F. y Margulies, N. (1969); Lawler, E. E.; Payne, R. L.; Pheysey, D. C. y Pugh, D. S. (1971); Pritchard, R. P. y Karasick, B. W. (1973); Hall, D. T. y Oldham, D. R. (1974); Gavin, J. F. (1975), y Steers, R. M. (1977). 
Cuadro 1. Competencias individuales y grupales

\section{INDIVIDUALES}

INICIATIVA - PROACTIVIDAD: predisposición a crear las condiciones que, si bien no aseguran el desarrollo de los acontecimientos, procuran disminuir o atenuar las circunstancias de naturaleza adversa a lo inicialmente proyectado.

ADAPTACIÓN - FLEXIBILIDAD: facultad por la que la persona es capaz de amoldarse al entorno, a la situación y/o a las circunstancias sin que ello genere contradicciones o conflictos entre sí mismo, el puesto que ocupa y el medio en el que ha de desenvolverse.

LIDERAZGO PERSONAL: capacidad para persuadir, orientar e influir emocional y técnicamente a los individuos buscando el desarrollo de sus conocimientos, capacidades y habilidades personales y profesionales.

ANÁLISIS DE PROBLEMAS - TOMA DE DECISIONES: habilidad relacionada con la evaluación de situaciones, enmarcadas en un contexto de litigio, donde se hace necesaria la solución del conflicto en tiempo y forma.

\section{GRUPALES}

COMUNICACIÓN EFECTIVA: habilidad que permite enviar, recibir y procesar datos e informaciones en un contexto interindividual, favoreciendo la adquisición, la estimulación y el desarrollo de los comportamientos grupales orientados hacia un fin determinado.

TRABAJO EN EQUIPO: desarrollo de las facultades interindividuales que permitan la consecución de resultados en orden a los objetivos propuestos por la organización, optimizando los medios humanos y técnicos puestos a su alcance.

NEGOCIACIÓN: conjunto de habilidades orientadas a la obtención del máximo provecho de los representados en situaciones de confrontación, en las que generalmente deben cederse posiciones buscando una contraprestación satisfactoria para las partes.

MOTIVACIÓN INTERGRUPAL: capacidad generadora de energía que permite el impulso y/o el ánimo necesario para conseguir los fines propuestos en el equipo de trabajo.

Fuente: elaboración propia.

de forma más pormenorizada de las siguientes cuestiones: cultura y organización formal existente, aspectos retributivos y expectativas motivacionales, comunicación y concentración del poder, relaciones intra e interpersonales en el trabajo y conciliación de la vida personal y profesional.

- Contenido del trabajo: toma como referencia aspectos tales como el entorno y el equipo de trabajo, el diseño de puestos y tareas, el dimensionamiento de plantillas y la carga de trabajo asociada. 
- Significado del grupo: planea sobre elementos que condicionan o determinan el significado y/o el grado de cohesión del grupo. Ejemplo de ello son los niveles de comunicación efectiva, la motivación intrapersonal y el liderazgo proactivo.

- Apreciación personal del individuo: se imbrica con todas aquellas percepciones objetivas y subjetivas - que, por extensión y en función de la frecuencia, pueden hacerse extensivas al grupo y al resto de la organizaciónentre las que se mencionan: el grado de conocimientos, el nivel de capacidades y la escala de habilidades sociales.

Ahora bien, y debido nuevamente a la dificultad teórico-práctica por mensurar este impacto, se hace clave trascender los citados escenarios representados - a través del análisis de las variables subyacentes - sin desatender, por otro lado, la atención al conjunto de sintomas asociados (ver cuadro 1), que no son sino expresión de las fuentes de conflicto tácitas o expresas de la degradación del clima laboral organizativo.

En este sentido, analizar este conjunto de síntomas permitirá al investigador social anticipar posibles vías de análisis, a partir de las cuales sea posible corregir o disminuir la erosión evidenciada en el clima laboral.

\section{Papel de la gestión por competencias en la gestión del clima laboral}

Desde que Mc Clelland (1973) aludiera explícitamente al término competen$\mathrm{cia}^{2}$ como aquello que realmente causa un rendimiento superior en el trabajo, es decir, aquellos elementos que inciden al alza sobre los resultados inicialmente esperados, largo ha sido el debate sobre su interpretación. Al hilo de esto comentarios, una aproximación al concepto hace pensar que las competencias pueden consistir en:

- Motivos: entendidos como la necesidad subyacente o la forma de pensar que impulsa, orienta y selecciona la conducta de una persona. Por ejemplo: la necesidad de un logro.

- Rasgos de carácter: predisposición general a comportarse o a reaccionar de un modo determinado. Ejemplo de ello son: la confianza en uno mismo, el autocontrol y la resistencia al estrés, por citar sólo algunos.

- Concepto sobre uno mismo: lo que uno piensa, lo que valora y/o lo que está interesado en realizar el sujeto, orientando consiguientemente su patrón de comportamiento individual.

- Conocimientos: lo que se sabe sobre una herramienta, una técnica, una ciencia y/o una habilidad, lo cual determina su capacidad de actuación y, por tanto, condiciona su actuación.

- Capacidades cognitivas y de conducta: ya sean ocultas (razonamiento deductivo) u observables (escucha activa).

2. Ver McClelland, D. (1973). «Testing for competence rather that intelligence». American Psychologist, 28 (I), p. 1-14. 


\section{Cuadro 2. Relacion entre el cuadro competencial y las dimensiones del clima laboral}

\begin{tabular}{|l|l|}
\hline \multicolumn{1}{|c|}{$\begin{array}{c}\text { Síntomas relacionados } \\
\text { con el contexto organizativo }\end{array}$} & \multicolumn{1}{c|}{ Variables subyacentes } \\
\hline $\begin{array}{l}\text { Deterioro de misión, vision y } \\
\text { valores junto a bajos niveles de } \\
\text { compromiso hacia la propia } \\
\text { organización e indefinición de } \\
\text { objetivos organizativos. }\end{array}$ & $\begin{array}{l}\text { Cultura y organización formal } \\
\text { existente. }\end{array}$ \\
\hline $\begin{array}{l}\text { Estancamiento de la promoción } \\
\text { profesional, salarios discutidos } \\
\text { internamente y con relación al } \\
\text { sector, precariedad laboral y } \\
\text { escaso reconocimiento del } \\
\text { estatus adquirido. }\end{array}$ & $\begin{array}{l}\text { Aspectos retributivos y } \\
\text { expectativas motivacionales. }\end{array}$ \\
\hline $\begin{array}{l}\text { Escasa participación en la toma } \\
\text { de decisiones y falta de control } \\
\text { sobre el trabajo. }\end{array}$ & $\begin{array}{l}\text { Comunicación y concentración } \\
\text { de poder. }\end{array}$ \\
\hline $\begin{array}{l}\text { Aislamiento físico, poca relación } \\
\text { con los superiores y los } \\
\text { colaboradores, junto a conflictos } \\
\text { interpersonales. }\end{array}$ & $\begin{array}{l}\text { Relaciones intrapersonales e } \\
\text { interpersonales en el trabajo. }\end{array}$ \\
\hline $\begin{array}{l}\text { Conflictos entre el trabajo y el } \\
\text { hogar, poco apoyo en el hogar, } \\
\text { problemas derivados de una } \\
\text { doble carrera. }\end{array}$ & $\begin{array}{l}\text { Conciliación entre la vida } \\
\text { personal y la profesional. }\end{array}$ \\
\hline
\end{tabular}

\begin{tabular}{|l|l|}
\hline \multicolumn{1}{|c|}{$\begin{array}{c}\text { Síntomas relacionados } \\
\text { con el contenido del trabajo }\end{array}$} & \multicolumn{1}{c|}{ Variables subyacentes } \\
\hline $\begin{array}{l}\text { Problemas relacionados con la } \\
\text { fiabilidad, la disponibilidad, la } \\
\text { idoneidad y el mantenimiento, } \\
\text { tanto del equipo como de las } \\
\text { instalaciones. }\end{array}$ & $\begin{array}{l}\text { Entorno del trabajo y equipo de } \\
\text { trabajo. }\end{array}$ \\
\hline $\begin{array}{l}\text { Falta de variedad, pérdida global } \\
\text { del proceso de trabajo, } \\
\text { fragmentacion y ausencia de } \\
\text { significado, infrautilización y } \\
\text { sobreutilización de las } \\
\text { cualificaciones. }\end{array}$ & Diseño de puestos y de tareas. \\
\hline $\begin{array}{l}\text { Sobrecarga o escasa carga de } \\
\text { trabajo, falta de control con } \\
\text { respecto al ritmo establecido, } \\
\text { altos niveles de presión en } \\
\text { tiempo y forma. }\end{array}$ & $\begin{array}{l}\text { Dimensionamiento de plantillas } \\
\text { y carga de trabajo asociada. }\end{array}$ \\
\hline
\end{tabular}

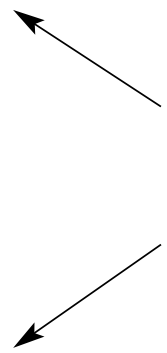

\begin{tabular}{|l|l|}
\hline \multicolumn{1}{|c|}{ Individuales } & \multicolumn{1}{c|}{ Grupales } \\
\hline Iniciativa y proactividad. & Comunicación efectiva. \\
\hline Adaptación y flexibilidad. & Trabajo en equipo. \\
\hline Liderazgo personal. & Negociación. \\
\hline $\begin{array}{l}\text { Análisis de problemas y } \\
\text { toma de decisiones. }\end{array}$ & Motivación intergrupal. \\
\hline
\end{tabular}

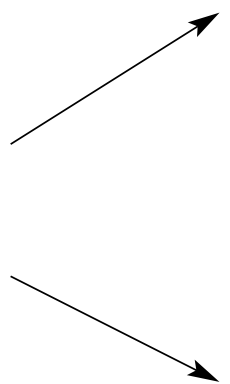

\begin{tabular}{|l|l|}
\hline \multicolumn{1}{|c|}{$\begin{array}{c}\text { Síntomas relacionados } \\
\text { con el significado del grupo }\end{array}$} & Variables subyacentes \\
\hline $\begin{array}{l}\text { Problemas relacionados con la } \\
\text { ausencia de comunicación por } \\
\text { parte del emisor, del receptor, de } \\
\text { los canales, de los mensajes y de } \\
\text { los contextos inadecuados. }\end{array}$ & Comunicación efectiva. \\
\hline $\begin{array}{l}\text { Ausencia de expectativas, bajos } \\
\text { niveles de reconocimiento y } \\
\text { proyección profesional en el } \\
\text { corto, medio y largo plazo. }\end{array}$ & Motivación intrapersonal. \\
\hline $\begin{array}{l}\text { Estilos de dirección autoritarios } \\
\text { basados en la fiscalización del } \\
\text { trabajo y la ausencia de planes } \\
\text { personalizados que impidan el } \\
\text { desarrollo de las personas. }\end{array}$ & Liderazgo proactivo. \\
\hline
\end{tabular}

\begin{tabular}{|l|l|}
\hline \multicolumn{1}{|c|}{$\begin{array}{c}\text { Síntomas relacionados } \\
\text { con la apreciación personal } \\
\text { del individuo }\end{array}$} & Variables subyacentes \\
\hline $\begin{array}{l}\text { Lagunas, errores, desorientación } \\
\text { y significado del valor aportado } \\
\text { al trabajo encomendado. }\end{array}$ & Grado de conocimientos. \\
\hline $\begin{array}{l}\text { Exceso de responsabilidad para } \\
\text { el puesto desempeñado o tareas } \\
\text { superadas para la potencialidad } \\
\text { teórica del individuo. }\end{array}$ & Nivel de capacidades. \\
\hline $\begin{array}{l}\text { Carencias de herramientas } \\
\text { intrapersonales e interpersonales } \\
\text { para el desarrollo de las } \\
\text { competencias del puesto en el } \\
\text { entorno de trabajo. }\end{array}$ & Escala de habilidades. \\
\hline
\end{tabular}

Fuente: elaboración propia. 
Buscando un nexo de unión con la propuesta trazada anteriormente, el modelo que, a continuación, se propone, describe a las competencias — siempre desde nuestra percepción particular - en dos bloques interrelacionados entre sí, las individuales y las grupales ${ }^{3}$, ambas concebidas como el "espíritu» que da sentido armonioso y coherente a la proyección personal y profesional del individuo en el conjunto de la organización.

De este modo, el cuadro 2 pone de manifiesto como las dimensiones individuales (iniciativa y proactividad; adaptación y flexibilidad; liderazgo personal; análisis de problemas y toma de decisiones) y las dimensiones grupales (comunicación efectiva; trabajo en equipo; negociación; motivación intergrupal), se convierten en los resortes que sustentan el modelo de clima laboral a través de los escenarios descritos (contexto organizativo, contenido del trabajo, significado del grupo y apreciación personal del individuo).

Este «efecto impulsor» — si se quiere ir más al detalle— explicaría también como un sistema de gestión por competencias congruente, permitiría reducir los síntomas de deterioro y, en consecuencia, una mejor modulación de las variables relacionadas.

\section{Conclusiones}

El clima es, en principio, una cuestión «ajena» a la tarea que esté realizando el individuo, dado que pueden observarse diferentes sensaciones de clima en personas que desarrollan el mismo trabajo, y es que la percepción que de la realidad albergan las personas, puede no ser consciente en muchos casos y, en otros, estar distorsionada por otra serie de elementos contaminantes.

El análisis del clima laboral debe concebirse, más que como un estado, cercado en el tiempo, como un proceso dinámico que varía en función de unas coordenadas espacio-temporales, sin que por esto pueda asegurarse una proyección del mismo, aún en el corto plazo. Por ello, desarrollar la capacidad de anticipación a situaciones adversas y comprometidas, permitirá amortiguar la degradación del mismo, mediante el tratamiento preventivo de los síntomas y, lo que es más importante, entender qué variables se encuentran como telón de fondo.

Por otro lado, la definición de un modelo de gestión por competencias ayuda a la construcción de una dinámica organizativa y de gestión de recursos humanos, explicitando los valores, los códigos éticos y deontológicos de quien se encuentra en la organización, dirigiendo sus comportamientos, remarcando lo que se espera de él y del grupo, lo que sin duda contribuye notablemente a la búsqueda de un clima laboral satisfactorio.

Nuestra aportación, a la vista de las argumentaciones anteriores, quiere enfatizar en la idea de que tanto el contexto organizativo, el contenido del trabajo, el significado del grupo como la apreciación personal del individuo (escenarios

3. Esta diferenciación, pretende evidenciar el mayor peso - individual o grupal- de determinadas competencias, siguiendo los planteamientos teóricos de Mc Clelland, D. (1973) y las acotaciones reseñadas por Bovatzis, R. E. (1982). 
del clima laboral) pueden modelarse en función de la construcción de un modelo de gestión por competencias, siendo éste quien construye, jerarquiza y moviliza las variables por las que una organización y, lo que es más importante, las personas, se desenvuelven. Este análisis polisémico permite, por otro lado, enriquecer el caudal semántico del diagnóstico y del tratamiento del clima laboral.

En este sentido - sobre la base de haber aclarado razonablemente los aspectos previos - cabe finalmente preguntarse si es posible poner en correspondencia el clima laboral de las organizaciones con la gestión por competencias. En otras palabras: ¿es factible mejorar el clima laboral como respuesta a un modelo de gestión por competencias? o, dicho de otro modo, ¿a través de las competencias, puede mejorarse el clima laboral?

Contestar afirmativamente a esta cuestión permite explorar en las relaciones causales anteriores. Por un lado, el buen o el mal clima laboral que puede «respirarse». una organización puede ayudar a redefinir y, en su caso, mejorar el marco sobre el que se inscribe un modelo de gestión por competencias y, por otro, un sistema de competencias bien construido permitirá mejorar la percepción del clima laboral existente. Es por ello que observar una retroalimentación en ambos sentidos y verificar lo biunívoco de los flujos trazados puede completar una visión mas integradora del análisis.

\section{Bibliografia}

BoyatZis, R. E. (1982). The competence manager. Nueva York: John Wiley \& Sons. ForeHAND, G.; GiLMER, B. (1964). «Environmental variation in studies of organizational behavior». Phychological Bulletin, 62, p. $361-382$.

FriedLANDER, F.; MARGULIES, N. (1969). «Multiple impacts of organizational climate and individual value system upon job satisfaction». Personnel Psychology, 22, p. 171-183.

GAVIN, J. F. (1975). «Organizational climate as a function of personal and organizational variables». Journal of Applied Psychology, 60, p. 135-139.

HalPin, A.; CROFTS, D. (1963). The organizational climate of schools. Chicago: University of Chicago.

Haro, J. M. de (2004). «¿Sabe alguien qué es una competencia?». Dirigir Personas, 30 , p. 8-17.

LAWLeR, E. E.; Hall, D. T.; Oldham, D. R. (1974). «Organizational climate: relations to organizational structure, process and performance». Organizational Behaviour and Human Performance, 11, p. 139-155.

Likert, R. (1967). The human organization. Nueva York: Mc Graw Hill.

LiTWIN, G.; STRINGER, R. (1968). Motivation and organizational climate. Boston: Harvard Business School.

MEYER, H. H. (1968). «Achievement motivation and industrial climates». En: TAGUIRI, R.; LiTWIN, G. H. (dirs.). Organizational climate; explorations of a concept. Boston: Harvard Business School, p. 35-65.

McClelland, D. (1973). «Testing for competence rather that intelligence». American Psychologist, 28 (I), p. 1-14. 
Payne, R. L.; Pheysey, D. C.; Pugh, D. S. (1971). «Organization structure, organizational climate and group structure: an explanatory study of their relations - ship in two British manufacturing companies». Occupational Psychology, 45, p. 45-56.

PRITCHARD, R. P.; KARASICK, B. W. (1973). "The effects of organizational climate on managerial job performance and job satisfaction». Organizational Behavior and Human Performance, 9, p. 126-146.

SCHNEIDER, B.; BARTLETT, C. J. (1968). «Individual differences and organizational climates: 1 . The research plan and questionnaire development». Personnel Psychology, 21, p. 447-455.

STEERS, R. M. (1977). Organizational effectiveness: a behavioral view. California: Goodyear Publishing. 